\title{
Phenotypic transformation and migration of adventitial cells following angioplasty
}

\author{
YONG-LI WANG, LI-ZHEN LIU, ZHONG-HUI HE, KUN-HONG DING and FENG XUE \\ Department of Interventional Radiology, Fengxian Center Hospital - Branch of Shanghai Sixth People's Hospital \\ Affiliated to Shanghai Jiaotong University, Shanghai 201400, P.R. China
}

Received February 8, 2012; Accepted March 16, 2012

DOI: $10.3892 /$ etm.2012.551

\begin{abstract}
The present study was designed to investigate the phenotypic transformation and migration of adventitial fibroblasts using 5-bromo-2'-deoxyuridine (BrdU) labeling following angioplasty and to explore the correlation between adventitial cells and post-angioplasty restenosis. A vascular restenosis model was established in 23 rats by injuring the common carotid artery with a wire. BrDU was used to label the fibroblasts followed by immunohistochemistry for $\alpha$-actin. Blood vessels were observed under light microscopy and scanning electron microscopy followed by image analysis. The number of BrDU-positive fibroblasts in the intima, media and adventitia of the blood vessels was determined 3, 7, 41 and 28 days after injury. The results demonstrated that at different time points, the number of BrDU-positive cells was significantly different in the intima, media and adventia $(\mathrm{P}<0.05)$. Electron microscopy indicated that the fibroblasts were full of cytoplasm. In addition, many secretory granules were noted on the rough endoplasmic reticulum and a large amount of microfilament bundles were noted after angioplasty. The fibroblasts transformed into myofibroblasts. Seven and 14 days after injury, the myofibroblasts formed wide pseudopods stretching to the fenestrae of the external and internal elastic lamina, and cells had a tendency to migrate into the lumen. The fibroblasts in the adventitia underwent transformation after percutaneous transluminal angioplasty and secreted $\alpha$-actin. In conclusion, the fibroblasts in the adventitia transformed into myofibroblasts, migrated into and proliferated in the intima and became a component of the newly generated intima. Adventitial cells are thus related to vascular restenosis.
\end{abstract}

Correspondence to: Dr Feng Xue, Department of Interventional Radiology, Fengxian Center Hospital - Branch of Shanghai Sixth People's Hospital, Affiliated to Shanghai Jiaotong University, 9588 Nanfeng Road, Shanghai 201400, P.R. China

E-mail: xuemd@yahoo.com.cn

Key words: angioplasty, vascular intima, myofibroblast, phenotype, migration

\section{Introduction}

The role of adventitial cells in restenosis after percutaneous transluminal angioplasty (PTA) has not been investigated and no related results were reported until the middle to late 1990 s (1-3). Upon arterial injury after PTA or intravascular stenting, the vascular adventitia is involved in the pathological repair or restenosis of blood vessels. Several studies have shown that restenosis following PTA involves the migration of not only vascular smooth muscle cells (VSMCs) in the media but cells of the vascular adventitia $(4,5)$. That is, fibroblasts in the vascular adventitia may migrate from the adventitia into the intima through the media, and the newly generated intima contains not only VSMCs but myofibroblasts that were transformed from fibroblasts in the adventitia $(6,7)$. However, the migration of cells in the adventitia has still not been confirmed in vascular repair following injury. Several studies have even yielded contradicting results, to the effect that the migration of fibroblasts from the adventitia into the media is absent $(8,9)$. A number of researchers have even postulated that a stem cell pool is present in the vascular adventitia. Following vascular injury, cells may produce the recall response that occurs in embryonic development. Non-muscle cells (stem cells in the adventitia) may transform into myofibroblasts and further into VSMCs (10). In the present study, the phenotypic transformation and migration of adventitial cells were observed and analyzed by morphological examination and detection of protein expression. Thus, we may provide evidence on the role of cell migration in vascular restenosis following PTA.

\section{Materials and methods}

Animal model of common carotid artery restenosis following PTA. We establishment an animal model of common carotid artery restenosis using a modified wire method. A total of 24 male SD rats weighing 250-300 g were divided into 4 groups and sacrificed at 3, 7, 14 and 28 days after vascular injury. For preparation of the arterial injury model, animals were intraperitoneally anesthetized with $10 \%$ chloral hydrate $(350 \mathrm{mg} / \mathrm{kg})$ and then a midline incision was made in the neck. Following separation of subcutaneous tissues, the bilateral common carotid artery (CCA) and external carotid artery (ECA) were exposed. A metal wire 0.038 inches in diameter was introduced into one of the CCAs through the ECA and 
the wire was forwarded and withdrawn 3-4 times to injure the vascular intima and all the layers of the blood vessels, which is similar to the vascular injury following balloon injury or intravascular stenting. The rats with injured CCAs served as the experimental group and those with intact CCAs were the control group.

Preparation of the antigen marker and labeling. 5-Bromo-2'deoxyuridine (BrdU) is involved in the synthesis of DNA in proliferating cells. After BrdU was integrated into proliferating adventitial cells, immunohistochemistry was performed to detect the number of BrdU-positive cells. First, the BrdU powder was dissolved in PBS at a final concentration of $25 \%$ $(0.25 \mathrm{mg} / \mathrm{ml})$. Then, pluronic biogum powder was mixed together with the above BrdU solution at a final concentration of $2.5 \%(2.5 \mathrm{mg} / \mathrm{ml})$. The injured and uninjured arteries were washed with sterilized normal saline and the liquid was removed. The BrdU solution was mixing by shaking before use, and then, $0.2 \mathrm{ml}$ of this solution was used to smear the bilateral CCAs.

Sample collection. Tissues were collected, embedded in paraffin and cut into $5-\mu \mathrm{m}$ consecutive sections which were adherent to the APES-treated slides. The sections were heated at $58-60^{\circ} \mathrm{C}$ for $30-60 \mathrm{~min}$ and the slides were allowed to cool to room temperature for further use.

Double immunohistochemistry for BrDU and $\alpha$-actin. Immunohistochemistry was performed using a traditional protocol. The sections were treated with BrDU monoclonal antibody to stain the proliferating and migrating cells in the different vascular layers. Following BrDU staining, these sections were treated with $\alpha$-actin monoclonal antibody for double immunohistochemistry. BrDU and BrDU monoclonal antibody were purchased from Sigma Chemical Co. and $\alpha$-actin monoclonal antibody, the ABC detection kit and DAB substrate kit were from Wuhan Boster Biotech Co., Ltd.

Determination of the number of BrDU-and/or a-actinpositive cells. Immunohistochemistry for BrDU was carried out alone and BrDU-positive cells displayed brown granules in the nucleus. Immunohistochemistry was also carried out for both BrDU and $\alpha$-actin, and the BrDU-positive cells had a dark blue nucleus while $\alpha$-actin-positive cells had brown cytoplasm. Cells positive for both BrDU and $\alpha$-actin displayed a dark blue nucleus and brown cytoplasm.

Counting of BrDU-positive cells. Four fields were selected at 4 quadrants of the blood vessel wall at a high magnification by 2 researchers blinded to the study details. The number of BrDU-positive cells in the vascular intima, media and adventitia were counted and the average eas calculated. The number of BrDU-positive cells in the blood vessels was the sum of these cells in the different vessel layers. The ratio of the number of BrDU-positive cells in each layer to the total number of BrDUpositive cells was calculated. This ratio was used to monitor changes in the number of positive cells in the blood vessels.

Electron microscopy. The 1- $\mathrm{mm}^{3}$ samples of tissues were fixed in $2.5 \%$ glutaraldehyde in $0.1 \mathrm{~mol} / 1$ sodium cacodylate buffer for $1 \mathrm{~h}$. After dehydration, embedding, sectioning and staining with uranyl acetate, the tissues were ultra-sectioned using an LKB-V ultramicrotome (Sweden) and the myofibroblasts in the different layers were observed under a transmission electron microscope (Hitachi H-7500, Japan).

Statistical analysis. Statistical analysis was carried out with SPSS version 10.0, and the t-test and analysis of variance were performed for comparisons. A value of $\mathrm{P}<0.05$ was considered to indicate statistical significance.

\section{Results}

Immunohistochemistry for BrDU. Three days after PTA, at high magnification, the number of adventitial cells increased and many cells had a brown nucleus indicative of proliferating cells. In the experimental group, the number of BrDU-positive cells was higher than that in the control group (cells having a brown nucleus were not found in the adventitia). In the media, an extremely small number of proliferating cells had a brown nucleus. In the media close to the lumen, cells with a brown nucleus were not present. Shedding of endothelial cells was noted but newly generated intima was absent. Seven days after PTA, the number of adventitial cells were markedly increased when compared with those 3 days after PTA, and the number of proliferating cells with a brown nucleus also increased. In the media, some proliferating cells had a brown nucleus and were distributed around the external elastic lamina. In the newly generated intima, a few new intimal cells with a brown nucleus were noted. Fourteen days after PTA, the number of adventitial cells was dramatically reduced when compared with the number of adventitial cells 3 and 7 days after PTA, and the number of proliferating cells displaying a brown nucleus also declined. In addition, many proliferating cells with a brown nucleus were noted in the media. In the relatively thick newly generated intima, a large number of proliferating cells with a brown nucleus was noted. At 28 days after PTA, only a few adventitial cells were found when compared with those 3, 7 and 14 days after PTA, and these cells were long-spindle-like while proliferating cells with a brown nucleus were not found. In the experimental group, the findings in the adventitia were similar to those in the control group. The media was thickened and proliferating cells with a brown nucleus were occasionally noted. The thick newly generated intima had a few proliferating cells with a brown nucleus (Fig. 1).

At high magnification, the cells in the different layers of the blood vessels were observed at different time points. Results showed that the number of BrDU-positive cells changed significantly in the experimental group. In the adventitia, median and intima, the number of BrDU-positive cells reached a maximal level at 7 and 14 days after PTA, respectively. The peak number of BrDU-positive cells in the different layers occurred alternatively in a chronological sequence (Fig. 1). Three and 28 days after PTA, the number of positive cells in the adventitia, media and intima was similar to those in the control group. At these two time points, proliferating cells (BrDU-positive) with a brown nucleus were rarely found. The number of BrDU-positive cells in the different layers at different time points is shown in Table I. 

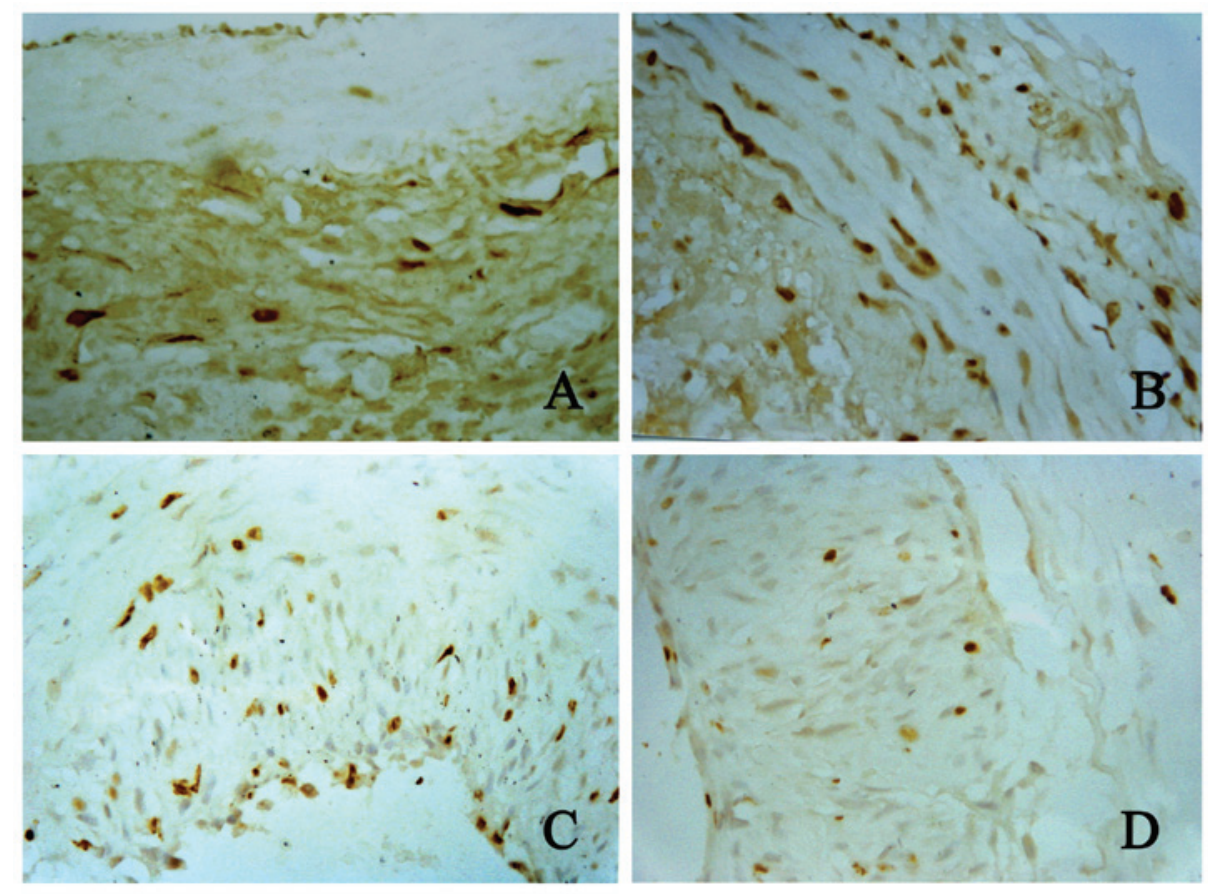

Figure 1. Detection of BrDU-positive cells by immunohistochemistry at different time points after PTA. (A) Three days after PTA, a few proliferating fibroblasts (brown cells) were positive for BrDU in the vascular adventitia; (B) 7 days after PTA, many BrDU-positive cells were noted in the adventitia and only a few BrDU-positive were noted in the media; (C) 14 days after PTA, the intima was thickened and the number of BrDU-positive cells increased and the nuclei of some cells were lightly stained; (D) 28 days after PTA, the thickening of the intima reduced and BrDU-positive cells were almost absent (magnification, x400).

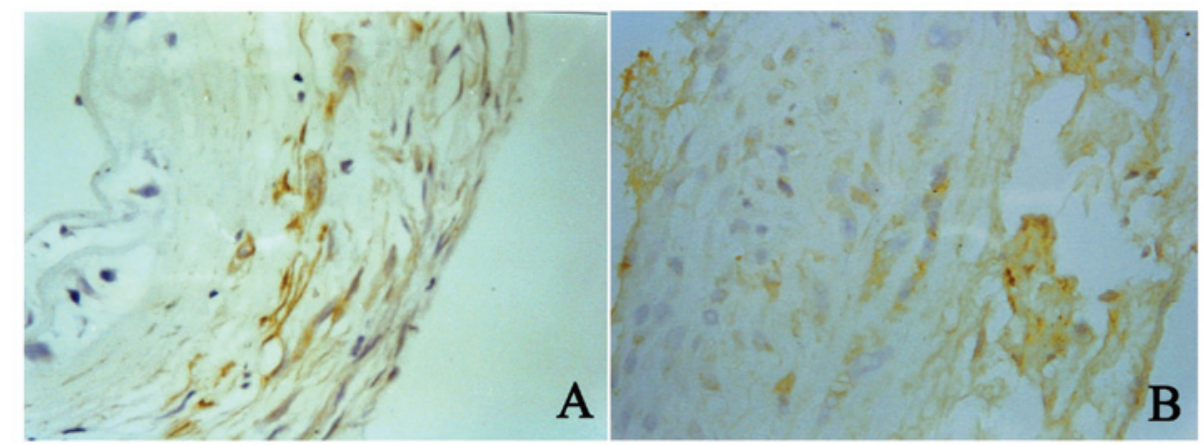

Figure 2. Double immunohistochemistry for BrDU and $\alpha$-actin to detect the migration and phenotypic transformation of fibroblasts in blood vessels at different time points. (A) Seven days after PTA, fibroblasts positive for both BrDU and $\alpha$-actin had a blue nucleus and brown cytoplasm. These cells aggregated around the external elastic lamina; (B) 14 days after PTA, cells positive for both BrDU and $\alpha$-actin were found in the intima (magnification, x400).

Double immunohistochemistry for BrDU and $\alpha$-actin. Seven days after PTA, the number of adventitial cells increased, the media was thickened and newly generated intima was barely noticeable. At 14 days after PTA, the intima was significantly thickened. Thus, in the experimental group, samples collected at 7 and 14 days were used for further double immunohistochemistry for BrDU and $\alpha$-actin. The nucleus was stained by BrDU and BrDU-positive cells had a blue nucleus. $\alpha$-actinpositive cells had brown cytoplasm. The results showed, 7 days after PTA, that the BrDU-positive cells in the adventitia were also positive for $\alpha$-actin, suggesting that the proliferating cells also expressed $\alpha$-actin. In addition, these cells aggregated around the external elastic lamina. At 14 days after PTA, the number of cells positive for both BrDU and $\alpha$-actin were markedly reduced but these cells in the newly generated intima increased dramatically (Fig. 2). Double immunohistochemistry revealed that the phenotype of the adventitial cells changed after PTA, and that the adventitial cells had transformed into myofibroblasts.

Electron microscopy. Three days after PTA, the fibroblasts in the adventitia exhibited a mature phenotype in the control group. The cells and nuclei were long-spindle-shaped and the cells had less pseudopod-like processes and less cytoplasm. After arterial injury, the spindle-shaped fibroblasts were found in the adventitia and the collagen and pseudopod-like processes increased. Seven days after PTA, cells with different shapes and sizes were found and the nuclei were irregular and spindle-like. These cells were noted to be myofibroblasts. These cells had processes with varied sizes and the number 
Table I. Number of BrDU-positive cells in the different vascular layers at different time points.

\begin{tabular}{|c|c|c|c|c|c|}
\hline \multirow[b]{2}{*}{ Layers } & \multicolumn{5}{|c|}{ BrDU-positive cells, mean \pm SD } \\
\hline & 3 days & 7 days & 14 days & 28 days & Control group \\
\hline Adventitia & $161.3 \pm 52.18$ & $247.2 \pm 33.72$ & $80.0 \pm 9.34$ & $4.3 \pm 1.97^{\mathrm{a}}$ & $2.1 \pm 2.99^{\mathrm{a}}$ \\
\hline Media & $8.8 \pm 4.96^{\mathrm{b}}$ & $52.8 \pm 25.20$ & $164.3 \pm 28.39$ & $8.2 \pm 4.79^{\mathrm{b}}$ & $1.3 \pm 8.944^{\mathrm{b}}$ \\
\hline Intima & $0.00 \pm 0.00^{c}$ & $165.3 \pm 2.86^{c}$ & $274.5 \pm 53.69$ & $45.5 \pm 13.78$ & $1.0 \pm 2.56^{\mathrm{c}}$ \\
\hline
\end{tabular}

ANOVA: Positive cells: ${ }^{a}$ in the adventitia, $\mathrm{P}<0.01$ comparisons among the different observed-time groups of 3,7 and 14 days, except for 28 days and control group $(\mathrm{P}=0.98)$; ${ }^{b}$ in the media, $\mathrm{P}<0.01$ comparisons among 3,7 and 14 days, except for 3,28 days and control group

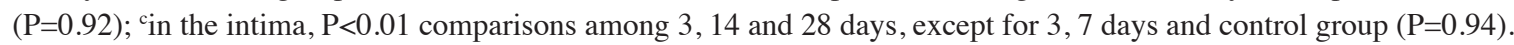

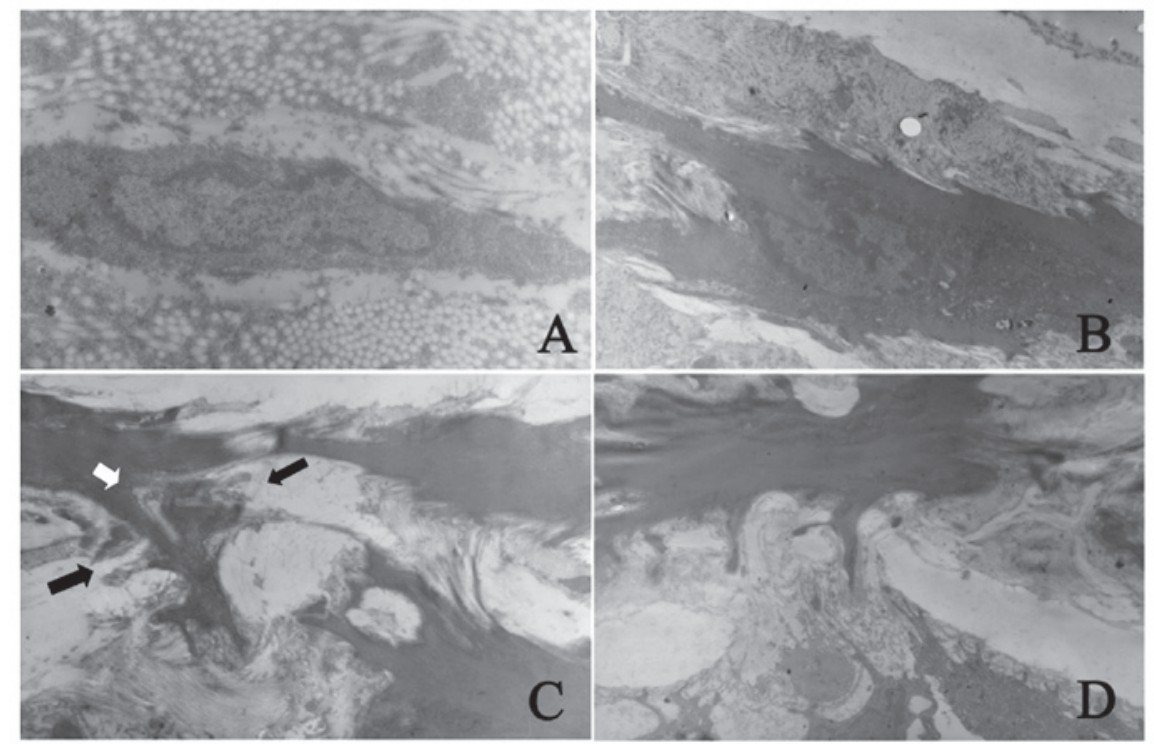

Figure 3. Detection of morphological changes and migration of fibroblasts after PTA by scanning electron microscopy. (A) The adventitial fibroblasts in normal blood vessels had a contractile quiescent phenotype. These cells had long-spindle nuclei but had less pseudopod-like processes (magnification, x15,000); (B) 7 days after PTA, the myofibroblasts in adventitia found processes with different sizes and the processes increased (magnification, x7,000); (C) 7 days after PTA, the myofibroblasts in adventitia formed a lot of processes and the lamellipodia (white arrow) crossed the fenestra of external elastic lamina (black arrow). Cells had a trend to migrate from adventitia into media (magnification, x10,000); (D) 14 days after PTA, the fenestra of internal elastic lamina enlarged and the myofibroblasts in the media formed several processes. The lamellipodia crossed the fenestra of internal elastic lamina (as in C). Cells had a trend to migrate from media into intima (magnification, x10,000).

of processes increased and formed lamellipodia stretching to the external elastic lamina and crossing the fenestra of the external elastic lamina. These cells had a tendency to migrate from the adventitia into the media. Fourteen days after PTA, the intima was markedly thickened and spindle-like and oval myofibroblasts were noted. The longitudinal axis of cell growth was vertical to the internal elastic lamina. Changes in the ultrastructures of the myofibroblasts were similar to those 7 days after PTA, but the distribution of intrinsic structures in the artery was different. At 14 days after PTA, the number of cells in the media and intima increased and the fenestra of internal elastic lamina enlarged. In the intima, myofibroblasts formed several processes and the lamellipodia stretched to and crossed the external elastic lamina. These cells had a tendency to migrate into the intima (Fig. 3). Twenty-eight days after PTA, the myofibroblasts in the different vascular layers transformed reversely into fibroblasts which were characterized by small nuclei, less nucleoplasm, small but obvious nucleoli and less cytoplasm. In addition, the number of pinocytotic vesicles was reduced and the lamellipodia almost disappeared in these cells.

\section{Discussion}

Cells in the adventitia are related to vascular restenosis following PTA and their characteristics are described. In mammals and humans, the vascular adventitia mainly consists of fibroblasts. Studies have shown that fibroblasts are involved in vascular repair following arterial injury. Soon after PTA, proliferating cells are present in the vascular adventitia or even in the media. These cells are large, spindle-like and similar to VSMCs but different from fibroblasts in functions (11). Patel et al found in an in vitro experiment that non-muscle cells in the vascular adventitia were highly adherent and proliferative 
and active in the synthesis of collagen. Thus, some researchers postulate that non-muscle cells in the adventitia are derived from fibroblasts and are also named myofibroblasts. However, the source and characteristics of myofibroblasts are still controversial. Immunohistochemistry has demonstrated that these cells synthesize and secrete $\alpha$-actin. $\alpha$-actin has been regarded as a specific marker of muscular cells. That is to say, the spindle-like cells which synthesize and secret $\alpha$-actin are muscular cells (MCs). Thus, myofibroblasts in blood vessels were previously referred to as VSMCs in different environments (12). However, Skalli et al (13) speculated that these cells are possibly derived from fibroblasts. In studies on myofibroblasts in human normal and pathological tissues, antibodies against desmin or vimentin or allogeneic different actin antibodies were used. Results revealed that general myofibroblasts express vimentin and cytoplasmic actin. Thus, researchers postulated that these cells were derived from fibroblasts. In pathological tissues (such as fibromatosis), the myofibroblasts have various characteristics: some express only vimentin (type $\mathrm{V}$ ) and some express both vimentin and $\alpha$-actin (type VA). Although components of the cytoskeleton are different, vimentin is consistently found in the cytoskeleton. These cells may have the same source (such as fibroblasts), and they express cytoskeletal proteins found in smooth muscles following specific stimulation (14). Thus, under normal conditions, fibroblasts express vimentin alone and do not express $\alpha$-actin or desmin. After arterial injury, fibroblasts in the adventitia were found to express $\alpha$-actin and their morphology was also altered. Scott et al defined these cells with changes in morphology in the adventitia and media by immunohistochemistry: myofibroblasts do not express desmin, caldesmon (a marker of well-differentiated VSMCs) and myosin but inductively express $\alpha$-actin (4). These characteristics suggest that myofibroblasts are different from VSMCs. However, both fibroblasts and myofibroblasts can express vimentin. Thus, we speculate that both cells have a common source: fibroblasts can transform into myofibroblasts. In the present study, a large amount of collagen bundle was found in the fibroblasts in the vascular adventitia under electron microscopy and the morphology was similar to that of fibroblasts. Cells had several processes, which were different from VSMCs. Immunohistochemistry indicated actin expression. Based on the findings of the morphological examination and immunohistochemistry, we can speculate that fibroblasts undergo phenotypic transformation and transform into myofibroblasts.

Phenotypic transformation of cells in the adventitia. A majority of researchers postulate that proliferating cells at an early stage following PTA are VSMCs in several regions of the vascular media. VSMCs have been found to proliferate at 24-48 $\mathrm{h}$ after vascular injury (15) and their proliferation reaches a peak at 7-14 days after injury. These proliferating cells are mainly found in the vascular media. Following PTA, VSMCs in the vascular media auto-regulate and the VSMC phenotype switches from a contractile quiescent to a proliferative motile phenotype (synthetic phenotype). These cells are star-like in morphology and organelles increased. However, these cells lack contraction while proliferation, migration and secretion are significantly enhanced. In vascular restenosis, the phenotype of VSMCs reverses approximately 2 weeks after arterial injury, and the VSMC phenotype switches from a proliferative motile to a contractile quiescent phenotype. At 4-6 months after injury, VSMCs are in a relatively quiescent state when the restenosis has formed (16). However, our findings were inconsistent with those described above. Under light microscopy, at 3-7 days after PTA, the cells in the vascular adventitia were significantly altered and a large amount of short-spindle-like fibroblasts were noted. They had large nuclei and full cytoplasm and were in a proliferative state. Analysis of BrDU-positive cells in all layers showed that cell proliferation was active in the vascular adventitia, which was consistent with the findings of Scott et al who observed that cell proliferation was mainly found in the adventitia at an early stage following PTA and the proliferation of adventitial cells was more obvious than those in the media. These findings differed from previous results that early cell proliferation was predominantly found in the vascular media. Double immunohistochemistry showed, 7 days after PTA, that the BrDU-positive cells began to express $\alpha$-actin. The myofibroblasts were transformed from fibroblasts located near the external elastic lamina. The $\alpha$-actin expression was also found in the newly generated intima 14 days after PTA. Twenty-eight days after PTA, the $\alpha$-actin expression was absent in the intima and adventitia. This suggests that cells migrating into the newly generated intima lose the ability to express $\alpha$-actin at a late stage following PTA and these cells were transformed into fibroblasts. Wilcox and Scott (5) found that, at an early stage after arterial injury, proliferating adventitial cells were negative for $\alpha$-actin. Phenotypic transformation occurred at 14 days after PTA and cells were positive for $\alpha$-actin. Our results indicated that the time when fibroblasts underwent phenotypic transformation and expressed $\alpha$-actin was different from that noted in the study of Wilcox and Scott. Our findings also confirmed that fibroblasts in the vascular adventitia underwent phenotypic transformation after arterial injury (6). The fibroblasts were transformed into myofibroblasts which possess the ability to proliferate, synthesize, migrate and specifically secrete and express $\alpha$-actin. Thus, not only VSMCs in the media but fibroblasts in the adventitia have throwback after PTA. That is to say, the phenotype of these cells becomes primitive (17), which is shared by fibroblasts and VSMCs and is also a premise of the proliferation and migration of adventitial cells. In the present study, as noted by electron microscopy, myofibroblasts had many mitochondria and active Golgi body and the granules on rough endoplasmic reticulum increased, which suggest that the cells acquired the functions of synthesis and secretion following phenotypic transformation.

Migration of adventitial cells. The migration of proliferating adventitial cells into the intima following arterial injury is still controversial (18-23). Our findings suggest that proliferating adventitial cells migrate into the intima and are the main source of newly generated intima. At 3 days after PTA, the proliferating cells were mainly found in the adventitia. Seven days after PTA, the number of proliferating cells in the adventitia reached a maximal level and these cells were mainly found around the external elastic lamina and the proliferating cells in the media began to increase. At 14 days after PTA, the proliferating cells in the adventitia reduced but those in the media and intima were markedly increased. The number of BrDU-positive cells in both 
the intima and media reached a peak at this time point and the thickness of the intima was maximized. At 28 days after PTA, the BrDU-positive cells in the adventitia, media and the newly generated intima were significantly reduced and the proliferating cells were almost absent in the adventitia and media. Analysis of the BrDU-positive cells in the different vascular layers showed that the number of BrDU-positive cells increased sequentially and alternatively: the increase in BrDU-positive cells in the media and newly generated intima occurred when the proliferating cells in the adventitia began to decrease. This may be explained by the fact that the myofibroblasts in the vascular adventitia migrated into the media and intima. These findings are consistent with those in a study by Scott et al. Smearing CCAs with BrDU solution can directly and effectively label myofibroblasts. Of note, in the present study, smearing CCAs with BrDU immediately after PTA only labeled the adventitial cells and labeling was not performed later. Thus, we must address the question of the origin of the the BrDU-positive cells in the newly generated intima 7-14 days after PTA. We speculate that the BrDU cells in the vascular adventitia proliferated and migrated from the adventitia into the intima, which is also supported by other studies (24). In addition, as noted at high magnification under a light microscope, a large amount of BrDU-positive cells in the newly generated intima with thickening were short-spindle-like and had full cytoplasm. These morphological features significantly resembled those of longspindle-like VSMCs in the media, and suggest that these cells are similar to myofibroblasts in the vascular adventitia. Results in other studies also support our findings; following VSMC activation under in vitro control, stimulation of the vascular adventitia could induce the migration of characteristic fibroblasts in the adventitia (25). Following transfection with a retrovirus carrying the $\beta$-galactosidase LacZ gene, primary fibroblasts in the adventitia were inoculated into the adventitia of rat CCAs to induce LacZ expression. In situ hybridization was employed to monitor the myofibroblasts. Results showed LacZ expression in the injured intima. In newly generated intima, the morphology of LacZ-positive fibroblasts was similar to adventitial cells indirectly labeled with BrDU. These findings indicate that the newly generated intima had cells derived from the adventitia $(26,27)$. In addition, we also observed findings similar to those in the study of Scott et al (4). The nuclei of BrDU-positive cells in adventitia, media and intima became light from 3 to 28 days after PTA. This implies that, from 3 days after PTA, the BrDU-labeled myofibroblasts in the adventitia migrated into the newly generated intima in which they underwent division, replication and proliferation. Thus, these cells lose BrDU-labeled DNA. These findings suggest that proliferating adventitial cells migrate from the adventia and cross the external elastic lamina and become the main cells in the newly generated intima. Additionally, electron microscopy showed, 7 days after PTA, that the myofibroblasts in the adventitia formed lamellipodia which stretched to the external elastic lamina and crossed the fenestra of the external elastic lamina. These cells had a tendency to migrate into the media. At 14 days after PTA, the fenestra of the internal elastic lamina in the media enlarged. The myofibroblasts formed lamellipodia with several processes which stretched to the internal elastic lamina and crossed the fenestra of the internal elastic lamina. These cells had a tendency to migrate into the intima.
Taken together, in vascular restenosis following PTA, adventitial fibroblasts undergo phenotypic transformation and migration. These cells migrate into the newly generated intima, transform into myofibroblasts and thus are involved in vascular restenosis.

\section{Acknowledgements}

Some of the results of the study were from a doctoral dissertation for Tianjin Medical University and we thank He N.S. for assistance with the research study.

\section{References}

1. Gutterman DD: Adventitia-dependent influences on vascular function. Am J Physiol 277: H1265-H1272, 1999.

2. Barker SG, Tilling LC, Miller GC, et al: The adventitia and atherogenesis: removal initiates intimal proliferation in the rabbit which regresses on generation of a 'neoadventitia'. Atherosclerosis 105: 131-144, 1994.

3. Schneider DB, Sassani AB, Vassalli G, Driscoll RM and Dichek DA: Adventitial delivery minimizes the proinflammatory effects of adenoviral vectors. J Vasc Surg 29: 543-550, 1999.

4. Scott NA, Cipolla GD, Ross CE, Dunn B, Martin FH, Simonet L and Wilcox JN: Identification of a potential role for the adventitia in vascular lesion formation after balloon overstretch injury of porcine coronary arteries. Circulation 93: 2178-2187, 1996.

5. Wilcox JN and Scott NA: Potential role of the adventitia in arteritis and atherosclerosis. Int J Cardiol 54 (Suppl): S21-S35, 1996.

6. Shi Y, O'Brien JE, Fard A, et al: Adventitial myofibroblasts contribute to neointimal formation in injured porcine coronary arteries. Circulation 94: 1655-1664, 1996.

7. Haurani MJ, Cifuentes ME, Shepard AD, et al: Nox4 oxidase overexpression specifically decreases endogenous Nox4 mRNA and inhibits angiotensin II-induced adventitial myofibroblast migration. Hypertension 52: 143-149, 2008.

8. Hecto DL, Jeremy DO, Kathy KG, et al: Adventitial cells do not contribute to neointimal mass after balloon angioplasty of the rat common carotid artery. Circulation 104: 1591-1593, 2001.

9. Fleenor BS and Bowles DK: Negligible contribution of coronary adventitial fibroblasts to neointimal formation following balloon angioplasty in swine. Am J Physiol Heart Circ Physiol 296: H1532-H1539, 2009.

10. Faggin E, Puato M, Zardo L, et al: Smooth muscle-specific SM22 protein is expressed in the adventitial cells of ballooninjured rabbit carotid artery. Arterioscler Thromb Vasc Biol 19: 1393-1404, 1999.

11. Patel S, Shi Y, Niculescu R, Chung EH, Martin JL and Zalewski A: Characteristics of coronary smooth muscle cells and adventitial fibroblasts. Circulation 101: 524-532, 2000.

12. Sipes JM, Guo N, Negre E, et al: Inhibition of fibronectin binding and fibronectin-mediated cell adhesion to collagen by a peptide from the second type I repeat of thrombospondin. J Cell Biol 121: 469-477, 1993.

13. Skalli O, Darby I and Gabbiani G: Smooth muscle actin is transiently expressed in by myofibroblasts during experimental wound healing. Lab Invest 63: 21-29, 1990.

14. Gabbiani G, Rungger-Brandle E, de Chastonay C, et al: Vimentincontaining smooth muscle cells in aortic intimal thickening after endotherlial injury. Lab Invest 20: 196-202, 1982.

15. Marnur JD, Rossikhina M, Guha A, Fyfe B, et al: Tissue factor is rapidly induced in arterial smooth muscle after balloon injury. $\mathrm{J}$ Clin Invest 91: 2253-2259, 1993.

16. Reidy MA, Fingerle J and Linddner V: Factors controlling the development of arterial lesions after injury. Circulation 86 (Suppl 6): III43-III46, 1992.

17. Hogeman B, Gillessen A, Bocker W, et al: Myofibroblast-like cells produce mRNA for type I and III procollagens in chronic active hepatitis. Scan J Gastroenterol 28: 591-594, 1993.

18. Wexberg P, Muck K, Windberger U, et al: Adventitial response to intravascular brachytherapy in a rabbit model of restenosis. Wien Klin Wochenschr 116: 190-195, 2004. 
19. Kingsley K, Huff JL, Rust WL, et al: ERK1/2 mediates PDGF-BB stimulated vascular smooth muscle cell proliferation and migration on laminin-5. Biochem Biophys Res Commun 293: 1000-1006, 2002.

20. Wang Z and Newman WH: Smooth muscle cell migration stimulated by interleukin 6 is associated with cytoskeletal reorganization. J Surg Res 111: 261-266, 2003.

21. Oparil S, Chen SJ, Chen YF, et al: Estrogen attenuates the adventitial contribution to neointima formation in injured rat carotid arteries. Cardiovasc Res 44: 608-614, 1999.

22. Mallawaarachchi CM, Weissberg PL and Siow RC: Smad7 gene transfer attenuates adventitial cell migration and vascular remodeling after balloon injury. Arterioscler Thromb Vasc Biol 25: 1383-1387, 2005 .

23. Torsney E, Hu Y and Xu Q: Adventitial progenitor cells contribute to arteriosclerosis. Trends Cardiovasc Med 15: 64-68, 2005.
24. Damrauer SM, Fisher MD, Wada H, et al: A20 inhibits postangioplasty restenosis by blocking macrophage trafficking and decreasing adventitial neovascularization. Atherosclerosis 211: 404-408, 2010

25. Li G, Chen YF, Greene GL, et al: Estrogen inhibits vascular smooth muscle cell-dependent adventitial fibroblast migration in vitro. Circulation 100: 1639-1645, 1999.

26. Li G, Chen SJ, Oparil S, Chen YF and Thompson JA: Direct in vivo evidence demonstrating neointimal migration of adventitial fibroblasts after ballooninjury of rat carotid arteries. Circulation 101: 1362-1365, 2000.

27. Appleby CE and Kingston PA: Gene therapy for restenosis - what now, what next? Curr Gene Ther 4: 153-182, 2004. 